This document is the accepted manuscript version of the following article:

Safin, A., Zweck, J., \& Minkoff, S. E. (2020). A one-way coupled model for the vibration of tuning fork-based trace gas sensors driven by a thermoacoustic wave. Applied Physics B:

Lasers and optics, 126(2), 29 (10 pp.). https://doi.org/10.1007/s00340-020-7376-8

\title{
A one-way coupled model for the vibration of tuning fork-based trace gas sensors driven by a thermoacoustic wave
}

\author{
Artur Safin $^{1,2}$, John Zweck ${ }^{1}$, Susan E. Minkoff ${ }^{1}$ \\ 1 Department of Mathematical Sciences, The University of Texas at Dallas, 800 W. Campbell Rd, Richardson, TX 75080, \\ USA. e-mail: artur.safin@eawag.ch, zweck@utdallas.edu, sminkoff@utdallas.edu \\ 2 Present address: Eawag: Swiss Federal Institute for Aquatic Science and Technology, Überlandstrasse 133, 8600 Dübendorf, \\ Switzerland.
}

The date of receipt and acceptance will be inserted by the editor

\begin{abstract}
We present a single computational model for both quartz-enhanced photoacoustic spectroscopy and resonant optothermoacoustic detection trace gas sensors. These sensors employ a quartz tuning fork to detect the acoustic pressure and thermal waves generated when a laser excites a trace gas. The model is based on a coupled system of equations developed by Morse and Ingard for pressure and temperature in a fluid. The pressure and temperature solutions drive the resonant vibration of the tuning fork, which is modeled using the equations of linear elasticity. At high ambient pressure, excellent agreement is obtained with laboratory experiments. This result provides the first quantitative match between a fully computational simulation and experiments for a QEPAS sensor. Such a model could ultimately facilitate sensor design optimization. At low ambient pressure (less than 60 Torr), quantitative agreement is obtained after re-weighting the contributions from the pressure and thermal components of the signal. While this result is a substantial improvement over previous results in which a scaling factor was required to obtain agreement at any ambient pressure, at low pressures it appears that a more accurate physical model may be required to match experimental data.
\end{abstract}

Key words trace gas sensors, optothermoacoustic detection, quartz-enhanced photoacoustic spectroscopy

This work was supported by the National Science Foundation under Grant No. DMS-1620293. The numerical simulations were performed on the Extreme Science and Engineering Discovery Environment (XSEDE), which is supported by National Science Foundation grant number ACI-1548562.

\section{Introduction}

Photoacoustic spectroscopy (PAS) is a well-established technique for trace gas detection. Applications of this method include environmental monitoring, disease diagnosis and the detection of toxic gases [1]. A quartz tuning fork (QTF) can be deployed as a transducer to detect the acoustic pressure wave generated by the excitation of the trace gas by a laser heat source, in a technique known as quartz enhanced photoacoustic spectroscopy (QEPAS) [2]. In addition to being compact and portable, QEPAS sensors are wavelength independent, nearly immune to background noise, and have a large linear dynamic range with the capability of detecting concentrations from a few percent to the parts-per-trillion range [3-6].

QEPAS sensors achieve optimal performance when the laser is focused near the top of the gap between the tines of the QTF. At low ambient pressure experiments show a significant contribution from a thermal wave that becomes dominant when the laser is focused near the bottom of the gap between the tines $[7,8]$. This method of detection is known as resonant optothermoacoustic detection (ROTADE). Because ROTADE sensors operate at low ambient pressure, viscous damping of the QTF is significantly reduced and the sensor offers greater wavelength selectivity due to the narrower absorption lines of the trace gas. Experiments have also been performed in which the acoustic and thermal contributions are both significant [7].

Current research into trace gas sensors seek to improve their sensitivity and selectivity through novel experimental setups and sensor modifications $[6,9,10]$. For example, the use of microresonator tubes $[4,11,12]$, custom QTF geometries [1,10], and dual QTF configurations $[5,13]$ has enabled significant improvements in sensor performance. The performance of these sensors could be further improved by using computational modeling to facilitate optimization of the geometry of the QTF. 
Petra et al. [14] used analytic solutions of the acoustic wave equation and the Euler-Bernoulli equation for a vibrating beam to predict the optimal position of the laser beam for a QEPAS system with a bare QTF. They obtained agreement to within a factor of about two with experimental results. Firebaugh et al. [15] used a finite element model to predict the optimal radial dimensions of two microresonator tubes used in a QEPAS sensor. Auost et al. [16] used a hybrid analytical-computational model for QEPAS sensors with microresonator tubes to compute the influence that the length of the microresonator tubes has on the resonance frequency and Qfactor of the system. However, they adjusted parameters in their model to obtain agreement with experimental data.

Modeling QTF-based sensors at lower ambient pressure is considerably more difficult. Due to the small scale of the QTF and the proximity of the laser to the material, the interaction between the acoustic and the thermal components of the process can significantly affect the signal generated by the sensor [17]. These interactions can be modeled by a coupled system of partial differential equations for the temperature, pressure, and velocity in a fluid due to Morse and Ingard [18], which generalize the classical acoustic pressure and heat equations. Kaderli et al. [19] derived an analytical solution of the pressure-temperature subsystem in a special case with cylindrical symmetry, and showed that at length scales on the order of $0.1 \mathrm{~mm}$, the temperature near the fluid-solid interface can be an order of magnitude larger than that computed using the heat equation alone. In our latest work [20], we developed a computational model to solve the same pressure-temperature equations in a fluid surrounding a QTF. We showed that in a parameter regime studied experimentally by Doty et al. [7], the pressure obtained by solving the Morse-Ingard equations predicts a smaller net force on the QTF than that obtained by solving the acoustic wave equation alone.

To model the detection of trace gases using QEPAS and ROTADE sensors, the Morse-Ingard equations need to be coupled to the equations of linear elasticity for the displacement of the QTF. In this paper, we propose a one-way coupled model in which the pressure and temperature of the fluid drive the deformation of the QTF. To add viscous damping to the model, we use experimentally measured values of the $Q$-factor of the QTF as an input parameter. We solve the equations in the model using the finite element method with the aid of a custom-designed preconditioner that facilitates the numerical solution of the resulting large linear system [20 $22]$. With this model we are able to handle both the QEPAS and ROTADE sensing modalities.

We compare the results of our numerical simulations to experimental results of Kosterev and Doty [7,23] who measured how the piezoelectric signal varies with respect to the position of the laser beam. These results were obtained with a bare QTF at both high and low ambient pressures. At high ambient pressure, we observe excellent agreement between the numerical and experimental results, with at most a $10 \%$ discrepancy in regions with strong signal. This result is the first published quantitative agreement between finite element simulations and experiments for a QEPAS sensor that does not require fitting any parameters (except for the $Q$-factor) or using normalization constants. At low ambient pressure, we obtained quantitative agreement with experiments by separately normalizing the contributions due to the acoustic and thermal components of the signal. This result suggests that there is a physical process that was omitted from our model but which is only important at low ambient pressures.

In Section 2, we describe our computational model. In Section 3, we compare the results from our numerical model to experimental data for both QEPAS and ROTADE sensors, and in Section 4 we discuss several physical mechanisms that could account for the discrepancy between the simulation and experimental results at low ambient pressure. Finally, in Section 5 we provide a summary of our results.

\section{Numerical model}

In this section we describe the one-way coupled model we developed for the pressure and temperature in the fluid and the induced elastic deformation of the QTF. This model, which is similar to the one developed by Petra et al. [24] in their modeling of ROTADE sensors, consists of the following three stages:

1. Determine the numerical eigenfrequency, $\omega_{0}$, of the QTF for the particular finite-element mesh;

2. Solve the pressure-temperature subsystem of the Morse-Ingard equations in the gas coupled to the heat equation in the QTF (with $\omega_{0}$ as an input parameter);

3. Compute the resulting deformation of the QTF using both $\omega_{0}$ and the pressure-temperature solution from stage 2 .

For brevity, we refer the reader to references [20,25] for the details of the finite element formulation of the model.

\subsection{Model for numerical eigenfrequency}

The tuning fork, which is cut from an anisotropic trigonal quartz crystal, resonates at $f_{0} \approx 2^{15} \mathrm{~Hz} \approx 32 \mathrm{kHz}$ in a vacuum $[4,26]$. Since QTFs have narrow resonance bandwidths [3], in laboratory experiments the precise value of the resonance frequency, $f_{0}$, must be measured when the sensor is calibrated. Likewise, with a computational model it is essential to precisely determine the 


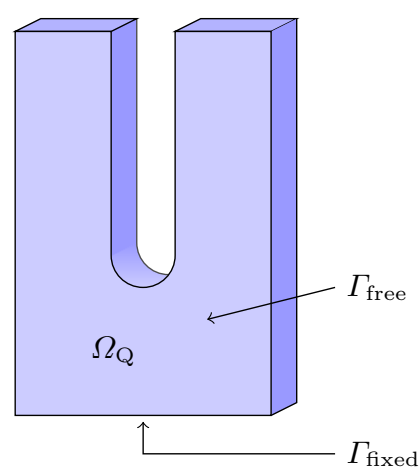

Fig. 1 The QTF, $\Omega_{Q}$, and its boundary. The fixed boundary, $\Gamma_{\text {fixed }}$, is the bottom surface of the QTF. The remaining faces, $\Gamma_{\text {free, }}$, are free to vibrate.

resonance frequency of the computational QTF, which exhibits a slight dependence on the particular finite element mesh that is employed to approximate the QTF geometry.

According to the theory of linear elasticity [27], the vibration of the QTF is governed by the equation of motion,

$$
\nabla \cdot \mathbf{C}[\mathbf{E}(\mathbf{u})]-\rho_{Q} \frac{\partial^{2} \mathbf{u}}{\partial t^{2}}=\mathbf{F},
$$

where $\mathbf{u}$ is the displacement of the QTF from its rest position, $\mathbf{C}$ is the fourth-order elasticity (stiffness) tensor that relates the strain, $\mathbf{E}(\mathbf{u})=\frac{1}{2}\left[\nabla \mathbf{u}+\nabla \mathbf{u}^{\mathrm{T}}\right]$, of the QTF to the stress applied to it, $\rho_{Q}$ is the density of quartz, and $\mathbf{F}$ models a body force. Since the motion of the tuning fork is time-harmonic, we assume that $\mathbf{u}(\mathbf{x}, t)=\Re e\left[\mathbf{u}(\mathbf{x}) e^{-i \omega t}\right]$. In this situation, (1) becomes

$$
\nabla \cdot \mathbf{C}[\mathbf{E}(\mathbf{u})]+\omega^{2} \rho_{Q} \mathbf{u}=\mathbf{F} .
$$

Since the QTF is secured at the base, we partition the boundary of the QTF into a subdomain, $\Gamma_{\text {fixed }}$, that is fixed and a subdomain, $\Gamma_{\text {free}}$, that is free to vibrate (see Fig. 1). Letting $\mathbf{n}$ denote the outward unit normal to the QTF, the resulting eigenproblem is

$$
\left\{\begin{array}{rlrl}
\nabla \cdot \mathbf{C}[\mathbf{E}(\mathbf{u})]+\rho_{Q} \omega_{0}^{2} \mathbf{u}=\mathbf{0} & \mathbf{x} \in \Omega_{\mathrm{Q}} \\
\mathbf{u}=\mathbf{0} & \mathbf{x} \in \Gamma_{\text {fixed }} \\
\mathbf{C}[\mathbf{E}(\mathbf{u})] \mathbf{n}=\mathbf{0} & \mathbf{x} \in \Gamma_{\text {free }}
\end{array}\right.
$$

where we have imposed a zero traction condition on $\Gamma_{\text {free }}[27]$. Here, $\omega_{0}=2 \pi f_{0}$ is the eigenfrequency to be determined.

We discretize the system using the finite element method with piecewise quadratic polynomials. For the solver, we use a Krylov-Schur eigensolver [28] with a shift-and-invert strategy available in the SLEPc package [29] and a direct solver to perform matrix inversion whenever necessary $[30,31]$. We then choose the eigenpair that corresponds to the mode in which the tines vibrate symmetrically in the $x z$-plane shown in Fig. 2 . We use the corresponding eigenfrequency, $\omega_{0}$, and the QTF mesh employed in the numerical simulation as inputs for the next two stages of the method.

\subsection{Model for the thermoacoustic wave}

The periodic heating of a trace gas by a laser generates a thermoacoustic wave in the fluid (gas) surrounding the QTF. Here, we model this process using a system of equations originally derived by Morse and Ingard [18] for perturbations in the acoustic pressure, $P$, and fluid temperature, $T_{F}$, given by

$$
\begin{aligned}
& \Delta P-\frac{\gamma}{c^{2}}\left(\frac{\partial^{2}}{\partial t^{2}}-\ell_{v} c \frac{\partial}{\partial t} \Delta\right)\left(P-\alpha T_{F}\right)=0, \\
& \ell_{h} c \Delta T_{F}-\frac{\partial}{\partial t}\left(T_{F}-\frac{\gamma-1}{\gamma \alpha} P\right)=-\frac{1}{\rho_{F} C_{p}} \frac{\partial S}{\partial t} .
\end{aligned}
$$

Here $\gamma$ is the isentropic expansion factor of the gas, $c$ is the speed of sound, $\alpha$ is the rate of change of pressure with respect to temperature at constant volume, $\rho_{F}$ is the density of the gas, $C_{p}$ is the specific heat. The quantities $\ell_{v}$ and $\ell_{h}$ are the characteristic lengths of viscosity and heat conductivity, respectively [18]. The forcing term, $S$, represents the heat power density deposited into the gas [32], which we model as a time-harmonic function of the form $S(\mathbf{x}, t)=\Re e\left[S(\mathbf{x}) e^{-i \omega_{0} t}\right]$, where the spatial dependence of the source is given by

$$
S(\mathbf{x})=\frac{\alpha_{\mathrm{eff}} W_{L}}{\pi w^{2}(y)} \exp \left[-\frac{2\left[\left(x-x_{s}\right)^{2}+\left(z-z_{s}\right)^{2}\right]}{w^{2}(y)}\right] .
$$

The constant $\alpha_{\text {eff }}$ is the effective absorption coefficient of the gas, and $W_{L}$ is the total laser power. The laser beam is aligned with the $y$-axis, and is focused at a point, $\left(x_{s}, y_{s}, z_{s}\right)$, that is halfway between the front and back faces of the QTF. The function $w(y)$ models the width of the laser beam. For QEPAS sensors [14], the beam width is modeled by $w(y)=\sigma$, and for ROTADE sensors it is given as in Petra [24] by

$$
w(y)=\sigma \sqrt{1+\left(\frac{y-y_{s}}{y_{R}}\right)^{2}}
$$

where $\sigma$ is the beam width at the focal point, and $y_{R}=$ $\pi \sigma^{2} / \lambda$ is the Rayleigh length. Here, $\lambda$ is the wavelength of the laser radiation [33].

As the source function is time harmonic, the thermoacoustic equations (4) reduce to the Helmholtz system

$$
\begin{aligned}
& \Delta P+\frac{\gamma}{c^{2}}\left(\omega_{0}^{2}-i \ell_{v} c \omega_{0} \Delta\right)\left(P-\alpha T_{F}\right)=0, \\
& \ell_{h} c \Delta T_{F}+i \omega_{0}\left(T_{F}-\frac{\gamma-1}{\gamma \alpha} P\right)=-\frac{1}{\rho_{F} C_{p}} S .
\end{aligned}
$$

We now describe the computational model we used to solve (7). We solve the system in a domain $\Omega=\Omega_{\mathrm{Q}} \cup \Omega_{\mathrm{F}}$, where $\Omega_{\mathrm{Q}}$ is the QTF domain and $\Omega_{\mathrm{F}}$ is the surrounding fluid (see Fig. 2). The laser is directed along the $y$ axis, and passes through the front and back faces, $\Gamma_{\mathrm{FB}}$, of the computational domain, which are parallel to the $x z$-plane. We let $\Gamma_{\text {side }}$ denote the side faces, which are 


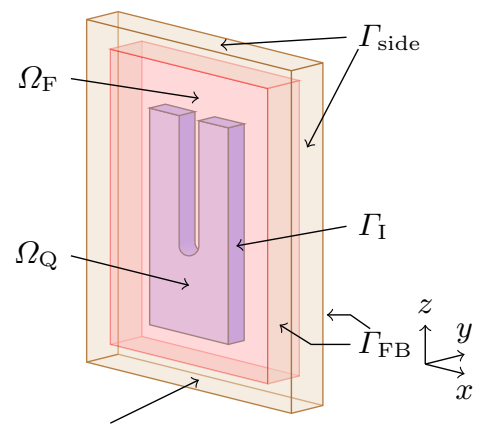

Absorbing boundary layer (PML)

Fig. 2 Domain for the trace gas sensor model. The tuning fork is shaded purple and the surrounding gas is colored pink. The absorbing boundary layer is shaded tan and is only implemented in the $x$ and $z$-directions.

perpendicular to the $x z$-plane, and $\Gamma_{\mathrm{I}}$ the interface between the tuning fork and the fluid. We denote the entire boundary of $\Omega_{\mathrm{F}}$ by $\Gamma=\Gamma_{\mathrm{I}} \cup \Gamma_{\text {side }} \cup \Gamma_{\mathrm{FB}}$.

Provided that the front and back faces of the computational domain are sufficiently far from the QTF, it is reasonable to impose zero Neumann boundary conditions for the pressure and temperature on $\Gamma_{\mathrm{FB}}$, since the thermoacoustic waves are largely independent of $y$ there. On the remaining exterior faces, $\Gamma_{\text {side }}$, of the domain (the tan region in Fig. 2), we implemented the perfectly matched layer (PML) method $[20,34,35]$ to attenuate the outgoing waves so that any portion that is reflected back into the computational domain is negligible.

For our one-way coupled model, we impose a zero Neumann boundary condition for the pressure on the QTF interface, $\Gamma_{\mathrm{I}}$, which models reflections of the pressure wave. For the temperature, we model the thermal dissipation into the tuning fork, $T_{Q}$, by solving the Helmholtz form of the heat equation,

$$
\nabla \cdot\left(\kappa_{Q} \nabla T_{Q}\right)+i \omega_{0} \rho_{Q} C_{p, Q} T_{Q}=0 \quad \text { in } \Omega_{\mathrm{Q}},
$$

where $\kappa_{Q}$ is the thermal conductivity tensor and $\rho_{Q}$ and $C_{p, Q}$ are the density and the specific heat of quartz, respectively. We couple the temperature variables in the fluid and QTF domains using the continuity of heat and heat flux conditions,

$$
\left\{\begin{array}{cl}
T_{F}(\mathbf{x})=T_{Q}(\mathbf{x}) & \text { (cont. of heat), } \\
\kappa\left(\nabla T_{F} \cdot \mathbf{n}\right)=\left(\boldsymbol{\kappa}_{Q} \nabla T_{Q}\right) \cdot \mathbf{n} & \text { (cont. of flux), }
\end{array}\right.
$$

where $\kappa_{F}$ is the thermal conductivity of the fluid.

As in our previous work [20], we discretize the pressure variable using piecewise linear polynomials. However since the temperature decays rapidly near the fluidstructure interface, we use piecewise quadratic polynomials to discretize temperature, which provides for a much more accurate solution. Once we solve the resulting linear system, we save the pressure solution on the free faces of the QTF, $\Gamma_{\text {free, }}$, and the temperature solution in the interior of the QTF to drive the vibration of the tuning fork.

\subsection{Model for the vibration of the tuning fork}

In the final stage of the model, we apply the solution of the thermoacoustic equations from the previous stage to determine the motion of the QTF. The heat that dissipates into the interior of the QTF induces a thermal stress in the structure which causes the tuning fork to vibrate. The pressure wave also drives the deformation of the QTF through the force it exerts on its boundary. The resulting motion of the tuning fork is governed by the differential equation (2). To incorporate damping into the model, we heuristically add an additional term, $-i \delta_{s} \omega_{0} \mathbf{u}$, as in Petra et al. [24]. The parameter, $\delta_{s}$, is given in terms of the $Q$-factor of the tuning fork by $\delta_{s}=\rho_{Q} \omega_{0} / Q$. The $Q$-factor quantifies how effectively the resonator resists energy losses due to damping. In this paper, we restrict attention to tuning forks for which the $Q$-factor has been measured in laboratory experiments. Recently, analytical methods have also been developed for determining the $Q$-factor of a QTF [36].

The stress tensor in a thermoelastic material is given by $\boldsymbol{\sigma}=\mathbf{C}[\mathbf{E}(\mathbf{u})]-\mathbf{C}\left[\boldsymbol{\alpha} T_{Q}\right]$ where $\boldsymbol{\alpha}=\operatorname{diag}\left(\alpha_{1}, \alpha_{2}, \alpha_{3}\right)$ is the thermal expansion tensor [37]. Incorporating the damping term, the deformation of the QTF driven by the thermoacoustic wave is therefore modeled using the equation

$$
\nabla \cdot \mathbf{C}[\mathbf{E}(\mathbf{u})]+\left(\rho_{Q} \omega_{0}^{2}-i \omega_{0} \delta_{s}\right) \mathbf{u}=\nabla \cdot \mathbf{C}\left[\boldsymbol{\alpha}_{s} T_{Q}\right],
$$

with the boundary conditions

$$
\left\{\begin{array}{cc}
\mathbf{u}=\mathbf{0} & \mathbf{x} \in \Gamma_{\text {fixed }}, \\
\nabla \cdot \mathbf{C}[\mathbf{E}(\mathbf{u})] \mathbf{n}=P \mathbf{n}+\mathbf{C}\left[\boldsymbol{\alpha}_{s} T_{Q}\right] \mathbf{n} & \mathbf{x} \in \Gamma_{\text {free }}
\end{array}\right.
$$

where $P$ and $T_{Q}$ are the solutions obtained in Section 2.2. The second equation in (11) is a stress continuity condition across the interface, $\Gamma_{\text {free }}$. To ensure that the numerical eigenfrequency, $\omega_{0}$, still corresponds to the numerical resonant mode of vibration, we use the same mesh and finite element discretization as in Section 2.1. We use a direct solver to determine the solution to the resulting finite element formulation of the system given by (10) and (11).

Finally, as in Petra et al. [24], we use an equivalence between the electrical and mechanical parameters of the QTF to convert the displacement into an electric signal,

$$
S=2 \nu \omega_{0} \mathbf{u}_{\mathrm{top}},
$$

where $\mathbf{u}_{\text {top }}$ is the displacement of the tip of the tine and the constant $\nu=7 \times 10^{-6} \mathrm{C} / \mathrm{m}$ is the effective piezoelectric coupling.

\section{Numerical results}

We now compare the results we obtained using the computational model to those obtained from laboratory experiments. The computations were performed in parallel 
Table 1 QTF parameters for the elasticity tensor, C, density of quartz, $\rho_{Q}$, and the thermal expansion tensor, $\boldsymbol{\alpha}_{s}$. The values are taken from Gautschi et al. [45].

\begin{tabular}{|c|r|}
\hline Const. & Value $\left[\mathrm{N} / \mathrm{m}^{2}\right]$ \\
\hline \hline$C_{11}$ & $86.80 \times 10^{9}$ \\
\hline$C_{12}$ & $7.04 \times 10^{9}$ \\
\hline$C_{13}$ & $11.91 \times 10^{9}$ \\
\hline$C_{14}$ & $-18.04 \times 10^{9}$ \\
\hline$C_{33}$ & $105.75 \times 10^{9}$ \\
\hline$C_{44}$ & $58.20 \times 10^{9}$ \\
\hline$C_{66}$ & $39.88 \times 10^{9}$ \\
\hline
\end{tabular}

\begin{tabular}{|c|c|}
\hline Const. & Value $\left[\mathrm{kg} / \mathrm{m}^{3}\right]$ \\
\hline \hline$\rho_{Q}$ & 2650.0 \\
\hline
\end{tabular}

\begin{tabular}{|c|c|}
\hline Const. & Value $[1 / \mathrm{K}]$ \\
\hline \hline$\alpha_{1}$ & $13.7 \times 10^{-6}$ \\
\hline$\alpha_{2}$ & $13.7 \times 10^{-6}$ \\
\hline$\alpha_{3}$ & $7.4 \times 10^{-6}$ \\
\hline
\end{tabular}

on the Stampede 2 cluster at the Texas Advanced Computing Center at The University of Texas at Austin. The finite element matrices were assembled using the deal. II package [38] and stored as distributed matrices and vectors in PETSc $[39,40]$. The mesh was generated using the Gmsh package [41] and partitioned for the parallel computation with p4est [42]. For post-processing, we used the Trilinos package [43]. For the sparse parallel LU solver we use MUMPS $[30,44]$, and for the generalized eigenproblem in stage 1 we use SLEPc [29].

The constants necessary to solve the eigenfrequency and the elastic deformation equations (2) and (10) are listed in Table 1, except for the values of the $Q$-factor which are given in the text below. We refer the reader to Safin et al. [20] for values of the geometric parameters of the QTF, the source parameters in (5), and the thermoacoustic parameters in (7) and (9). The only constants in the model we know of that depend on the ambient pressure, $P_{0}$, are the fluid density, $\rho_{F}$, which is proportional to $P_{0}$, the $Q$-factor which is inversely proportional to the square root of $P_{0}$, and the effective absorption constant, $\alpha_{\text {eff }}$, whose dependence on $P_{0}$ is discussed below.

\subsection{Results at high ambient pressure}

For sufficiently large ambient pressure, $P_{0}>50$ Torr, the detected signal is primarily due to the acoustic wave, corresponding to the QEPAS mode of detection. In this situation, Kosterev and Tittel [23] showed that the signal is largest when the laser is placed near the top of the QTF.

We compare the results obtained using the computational model to experimental data from Petra et al. [14], which was measured at an ambient pressure of $P_{0}=$ 450 Torr using a gas mixture of $1000 \mathrm{ppmv} \mathrm{NH}_{3}$ in $\mathrm{N}_{2}$. The $Q$-factor of the tuning fork was measured to be $Q=16,064$. The position of the laser beam was varied along a vertical line halfway between the tines of the QTF, and was measured from the base of the semicircular portion of the QTF. In Fig. 3.1 we show the amplitude (top) and phase (bottom) of the electric signal as a function of the height of the laser beam. The numerically computed signal is shown with a thick blue line, and the experimental data is shown with a thin red line. For reference, we also show the location of the top of the QTF using a dashed black line. The computed amplitude plot is largely in agreement with the experimental data, with at most a $10 \%$ discrepancy in the region where the signal is large. This result is an improvement over the analytic model of of Petra et al. [14] and the finite element model of Firebaugh et al. [46] for methane gas at 375 Torr, with both of these results being off by multiplicative factors greater than 2.1. The computed phase also agrees with experimental data where the signal is large. However, as in Petra et al. [14], near the base of the tuning fork and above the tuning fork the numerical results do not predict the sharp variations in the phase of the signal.

There are two mechanisms by which the QTF influences the acoustic pressure wave: reflections of the pressure wave off of the walls of the QTF, which we model using a Neumann boundary condition, and re-radiation of acoustic waves due to the QTF vibration, which we did not include in our one-way coupled model. In general, two-way coupling is required to model the forcing of the acoustic pressure by the QTF. Since two-way coupled models are computationally intensive and do not easily admit analytical solutions, in their work Firebaugh et al. [15,46] and Aoust et al. [16] approximated the reradiation from the QTF using an acoustic quadrupole source. However they did not demonstrate the necessity of this enhancement to the model. The good agreement we obtained with experiments suggests that acoustic reradiation is not a significant effect for QEPAS sensors operating at high ambient pressure.

\subsection{Results at low ambient pressure}

Next, we describe the results we obtained at low ambient pressure. In this regime, whether the thermal or the acoustic wave contributes more to the vibration of the QTF depends primarily on the position of the laser beam. If the laser is focused near the top between the tines of the QTF, then the acoustic wave is still the primary component of the electric signal [7]. But when the laser is focused near the bottom of the gap, the vibration is primarily driven by the thermal wave. We will compare the results we obtained from the computational model to an experimental data set measured by Kosterev and Doty [7] using a mixture of $0.5 \%$ acetylene in nitrogen $\left(\mathrm{C}_{2} \mathrm{H}_{2}: \mathrm{N}_{2}\right)$ at ambient pressures in the range $P_{0}=5-60$ Torr. At 5 Torr the measured $Q$-factor was $Q=79,794$, and at an arbitrary ambient pressure, $P_{0}$, we used the approximation $Q \approx 178,425 / \sqrt{P_{0}}[4,24]$.

The pressure and the temperature waves drive the motion of the QTF in a manner that is effectively independent of each other. Therefore, it is appropriate to treat the vibration of the QTF due to pressure as being entirely separate from the one due to temperature. As 

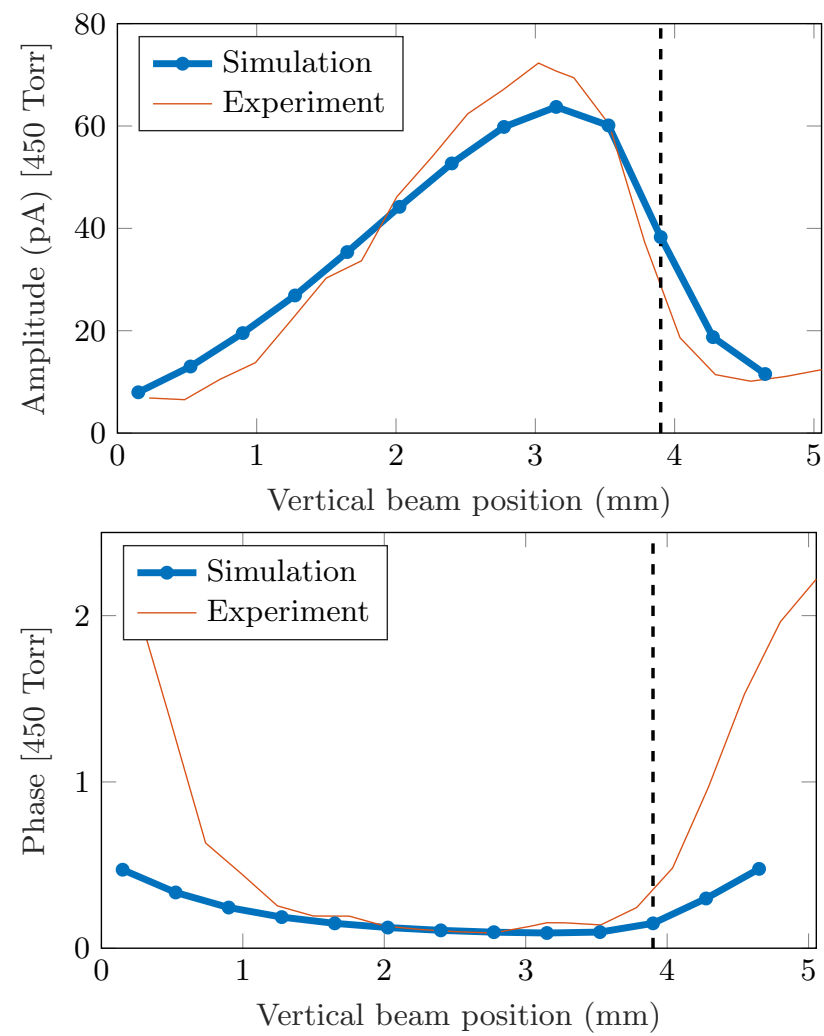

Fig. 3 Top: amplitude of the piezoelectric signal as a function of laser beam height for 1000 ppmv $\mathrm{NH}_{3}: \mathrm{N}_{2}$ at 450 Torr. Bottom: phase of the signal. The dotted black line corresponds to the top of the QTF.

a result, by selectively applying which forcing terms are used in equations (10) and (11), we can determine an acoustic component, $S_{\mathrm{P}}$, and a thermal component, $S_{\mathrm{T}}$, such that the overall signal, $S$, is just their direct sum, $S=S_{\mathrm{P}}+S_{\mathrm{T}}$. Our initial computational results (shown in Fig. 4) verify that the thermal component, $S_{\mathrm{T}}$, dominates over the acoustic component, $S_{\mathrm{P}}$, when the laser beam is located close to the base of the QTF.

To achieve quantitative agreement with the experimental results at low ambient pressure, we reweighted the contributions due to the acoustic and thermal components. We suppose that there are complex constants, $\beta_{g}$ and $\beta_{T}$, so that the electric signal is of the form

$$
S_{\text {nor }}(z)=\beta_{\mathrm{g}}\left[S_{\mathrm{P}}(z)+\beta_{\mathrm{T}} S_{\mathrm{T}}(z)\right],
$$

where $z$ is the vertical position of the laser beam and $S_{\text {nor }}$ is the normalized signal. We emphasize that the parameters, $\beta_{g}$ and $\beta_{T}$, only depend on the ambient pressure, $P_{0}$. At each ambient pressure, we estimate $\beta_{g}$ and $\beta_{T}$ using a least squares fit to the experimental data, $S_{\exp }$,

$\beta_{\mathrm{g}}, \beta_{\mathrm{T}}=\underset{\beta_{\mathrm{g}}, \beta_{\mathrm{T}}}{\arg \min }\left|\int_{I} \beta_{\mathrm{g}}\left[S_{\mathrm{P}}(z)+\beta_{\mathrm{T}} S_{\mathrm{T}}(z)\right]-S_{\exp }(z) d z\right|$,

where $I=\left[0, z_{\mathrm{top}}\right]$ is the set of laser beam positions in the experiment. The need for this post-facto modifica-
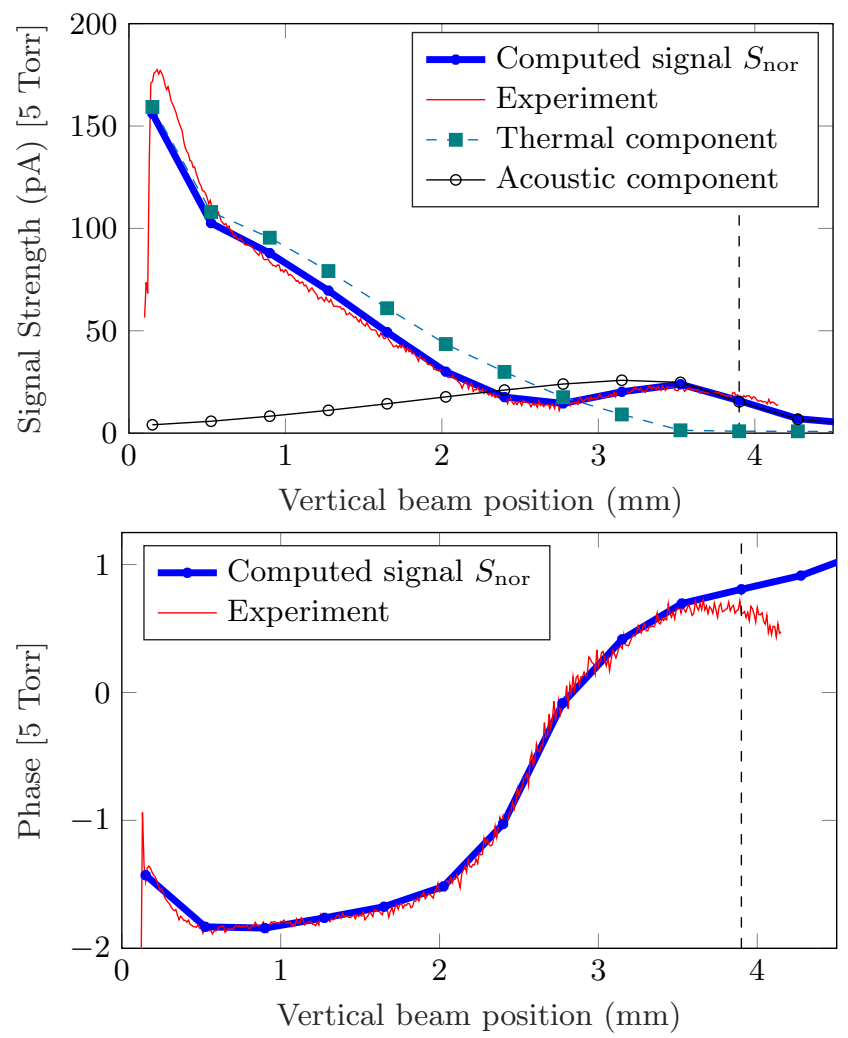

Fig. 4 Top: amplitude of the electric signal as a function of laser beam height for an experiment at 5 Torr. The plot also shows the thermal and acoustic components of $S_{\text {nor }}$. Bottom: phase of the signal. The dotted black line shows the position of the top of the QTF.

tion suggests that the acoustic and the thermal components contribute in different proportions than predicted by the model and that there is an additional phase shift between the pressure and temperature signals that needs to be accounted for.

A major reason we need to introduce the constant, $\beta_{g}$, is that we do not have a reliable method for determining how the effective absorption coefficient, $\alpha_{\text {eff }}$, in the laser source (5) depends on the ambient pressure, $P_{0}$. In particular, $\alpha_{\text {eff }}$, depends on the modulation amplitude and the absorption spectrum of the gas [14], and it is not simple to determine how these quantities depend on $P_{0}$. For this reason, our primary focus is on the parameter $\beta_{\mathrm{T}}$, which we allow to be complex-valued to account for a phase lag.

In Fig. 4, we compare the normalized signal, $S_{\text {nor }}$, (thick blue line) that we obtain using the model to the experimental data (red line) at an ambient pressure of 5 Torr. We show the amplitude in the top panel and the phase in the bottom panel. In the top panel we also show the amplitude of the acoustic component (solid black line with circles) and the thermal component (dashed green line with squares) of $S_{\text {nor }}$. We obtained good agreement between the experiment and the normalized signal across the entire range of beam positions, both in amplitude 

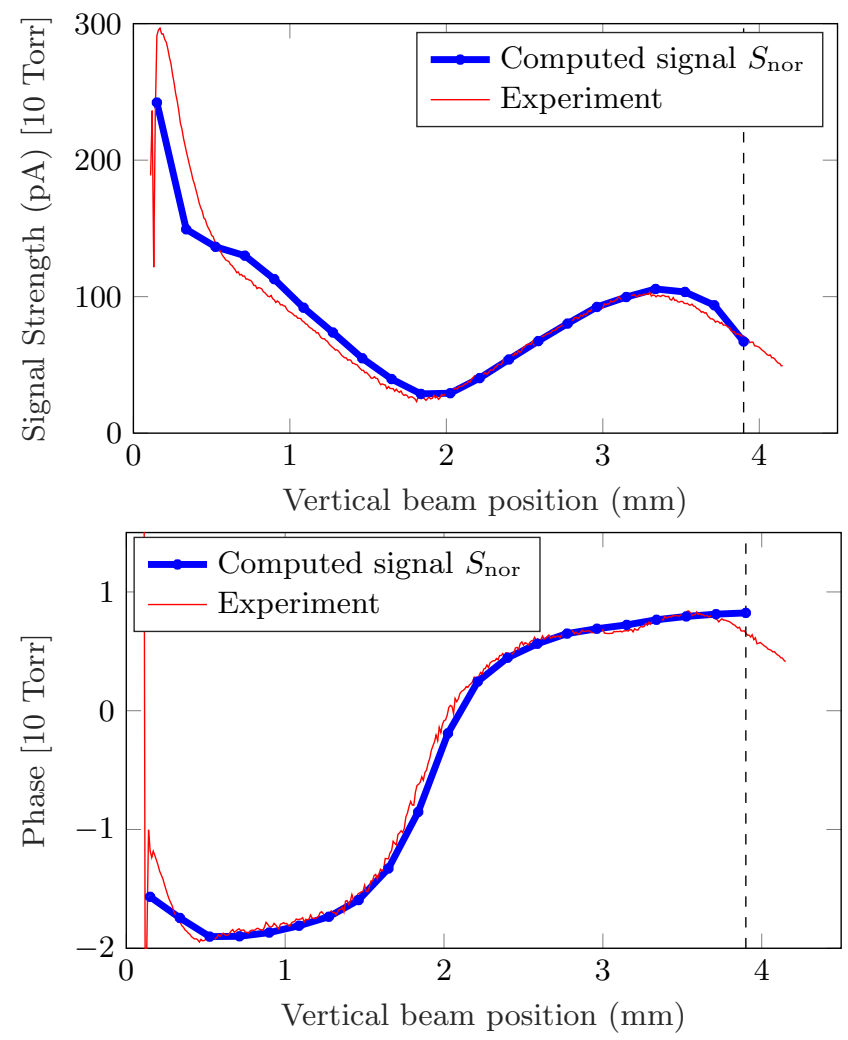

Fig. 5 Top: amplitude of the signal for an experiment at 10 Torr. Bottom: phase of the signal.

Table 2 Parameters $\beta_{\mathrm{g}}$ and $\beta_{T}$ obtained using the least squares fitting formula for different values of the ambient pressure $P_{0}$.

\begin{tabular}{|c|c|c|c|}
\hline$P_{0}$ (Torr) & $\left|\beta_{\mathrm{g}}\right|$ & $\left|\beta_{T}\right|$ & $\theta_{T}=\arg \left(\beta_{T}\right)$ \\
\hline 5 & 1.7417 & 17.6888 & 0.6360 \\
\hline 10 & 10.5503 & 9.0820 & 0.1311 \\
\hline 20 & 70.9690 & 4.3672 & -0.5461 \\
\hline 40 & 233.4987 & 4.0476 & -1.3266 \\
\hline 60 & 399.6060 & 4.4384 & -1.8445 \\
\hline
\end{tabular}

and phase. In Fig. 5 we compare the results from our simulations to the experimental data when the ambient pressure is 10 Torr. Once again, we obtain close agreement between the normalized signal from the simulations and the experimental data, which strongly suggests that the parameter, $\beta_{\mathrm{T}}$, reflects a missing component in the model. In Section 4 we discuss the modifications to the model we have investigated to ameliorate this discrepancy between our computational simulations and laboratory experiments at low pressures. In Table 2, we show the computed values of $\left|\beta_{\mathrm{g}}\right|$ and $\beta_{\mathrm{T}}$ at several values of $P_{0}$. Since interactions between the trace gas and the laser increase as the ambient pressure increases, we expect the effective absorption coefficient, $\alpha_{\text {eff }}$, to increase with $P_{0}$. However, since we used a fixed value of $\alpha_{\text {eff }}$ for all our low ambient pressure simulations, this increase is instead

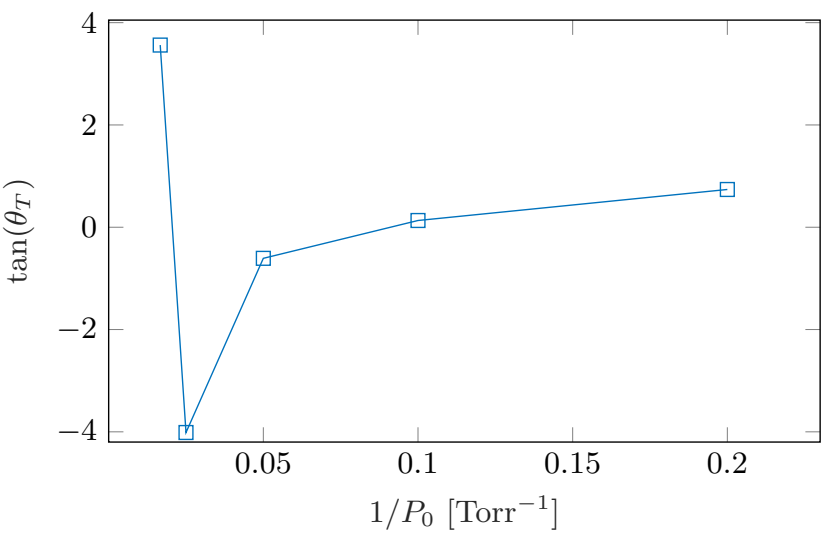

Fig. 6 Tangent of the phase $\operatorname{lag} \theta_{T}=\arg \left(\beta_{T}\right)$ as an inverse function of ambient pressure $P_{0}$.

reflected in $\left|\beta_{\mathrm{g}}\right|$. On the other hand, the magnitude of the parameter, $\beta_{\mathrm{T}}$, which represents the relative weighting of the thermal and acoustic components of the signal converges to a value of about 4 .

Finally, in the last column of the table we show the estimated phase lag, $\theta_{T}$, which accounts for the difference in time (on the order of microseconds) between the acoustic and the thermal components of the signal. The presence of this phase lag can be partially attributed to the fact that vibrational-to-translational (V-T) relaxation processes do not occur instantaneously, resulting in a small temporal delay in the generation of the pressure wave. In the case of a two-level molecular system, the phase lag due to $\mathrm{V}$-T relaxation satisfies [47]

$$
\tan \left(\theta_{T}\right) \propto \frac{1}{P_{0}}
$$

In Fig. 6, we test this proportionality relationship for the data in Table 2. Clearly, there is no linear relationship in the plot. However, the two leftmost values which correspond to pressures of 40 and 60 Torr may be unreliable since in these cases the thermal component of the signal is relatively small except near the bottom of the gap between the tines of the QTF. If we discard those two values, then the remaining points appear to be nearly linear.

Finally, we study how the strength of the normalized signal depends on the $(x, z)$-position of the laser beam for an experiment at 20 Torr. For each laser position in our simulation, we obtain the thermal and acoustic components of the piezoelectric current, $S_{T}$ and $S_{P}$, and use the estimated parameters in Table 2 to obtain the normalized signal $S_{\text {nor }}$. We show the results in Fig. 7, where the domain of study is $[-0.12,0.12] \mathrm{mm} \times[0.15,3.9] \mathrm{mm}$. Darker colors correspond to a stronger signal, and the thick black curve shows the outline of the QTF. We observe two regions with strong signal: near the bottom $(z<0.5 \mathrm{~mm})$ which corresponds to ROTADE sensing, and near the top $(z>2.5 \mathrm{~mm})$ where the QEPAS signal dominates. In the vicinity of the points with $(x, z)$ - 
Amplitude of Signal $(\mu \mathrm{A})[20$ Torr $]$

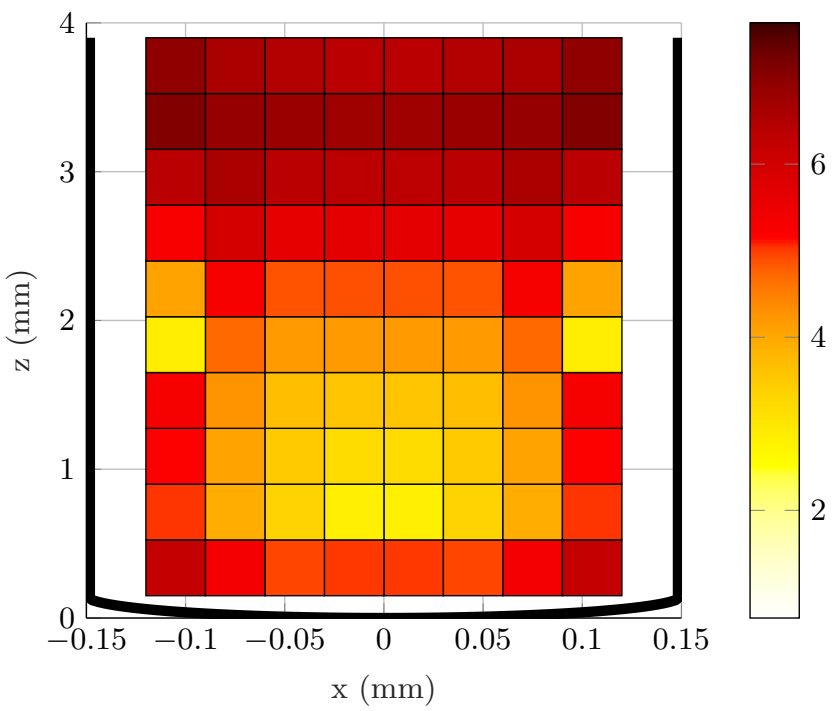

Fig. 7 Computed signal strength as a function of the $\left(x_{s}, z_{s}\right)$-position of the laser.

coordinates given by $(0,0.75)$ and $( \pm 0.1,1.75)$, we observe extremely weak signals due to the interference between the QEPAS and ROTADE sensing modalities. We note that our results are similar to the ones presented experimentally in Kosterev et al. [7], who also studied variations in the signal strength due to the $(x, z)$-position of the laser beam. Their experiment was also conducted at 20 Torr but for $\mathrm{CO}_{2}$ rather than the acetylene in nitrogen mixture we used.

\section{Discussion}

In this section, we discuss several physical mechanisms that could account for the need to reweight the pressure and temperature components of the signal at low ambient pressures.

First, as we stated above, we do not have a reliable method to predict how the effective absorption coefficient, $\alpha_{\text {eff }}$, depends on the ambient pressure. However, by itself this omission does not account for the phase difference between the pressure and temperature components. Motivated by the linear trend of the rightmost three values in the plot shown in Fig. 6, we developed a model that accounts for the relaxation time required for vibrationally excited molecules to return to the ground state and release their excess energy in the form of heat [25]. We added a third equation to the pressure-temperature system to model the energy density of thermally excited molecules in a two-level system, and their decay to the ground state due to collisions with other molecules and the walls of the QTF. However, for parameters corresponding to the acetylene-in-nitrogen experiment, this modification did not make a substantial improvement to the results.
Although we modeled the effect that the temperature has on the QTF displacement, it may also be necessary to account for how the stress induced in the QTF affects the temperature, as is described in the theory of linear thermoelasticity [37]. The phase discrepancy between the temperature and pressure signals at low ambient pressure might be rectified with a different fluid-structure interface conditon on the boundary of the QTF. One option would be to employ a two-way coupled model in which the QTF vibration forces the fluid velocity. In such a model, the full Morse-Ingard equations should also be used to relate the fluid velocity to the acoustic pressure and temperature. However, significant further model development is required to determine whether any of these models can explain the discrepancies we observed at low ambient pressure.

\section{Conclusions}

In this paper, we developed a three-stage computational model to determine the electric signal generated by a QEPAS or a ROTADE sensor. We implemented a oneway coupling algorithm in which the motion of the tuning fork is driven by the excitation of the trace gas by the laser. We modeled the generation of thermoacoustic waves using the Helmholtz form of a coupled system of partial differential equations derived by Morse and Ingard for a visco-thermo-acoustic fluid. We then used the equations of linear elasticity to compute the resulting vibration of the tuning fork, where we used the pressure on the walls of the QTF and the heating of the quartz material as forcing terms. We added an extra term to the equation of motion to account for damping that depends on experimentally measured values of the $Q$-factor.

When the sensor was operated at high ambient pressure we obtained excellent agreement between the computational model and experimental data. On the other hand, at low ambient pressure, the results of the simulations do not match the data. However, using a complexvalued reweighting of the thermal and acoustic components of the signal, we obtained good agreement with the experimental data, both in amplitude and phase. Using this normalized signal we studied how the signal strength depends on the laser beam position, and obtained good qualitative agreement with the data shown in Kosterev and Doty [7].

Taken together, our results suggest that there is a pressure-dependent physical mechanism that was omitted from our model and which only plays a role at low ambient pressure. Nonetheless, this paper presents an advance in the modeling both QEPAS and ROTADE trace gas sensors which is fundamental to optimizing their design.

Acknowledgments We thank Anatoliy Kosterev for sharing his expertise and for providing the experimental data sets. We also thank Noemi Petra for helpful discussions. 


\section{References}

1. P. Patimisco, G. Scamarcio, F. K. Tittel, and V. Spagnolo, "Quartz-enhanced photoacoustic spectroscopy: A review," Sensors, vol. 14, no. 4, pp. 6165-6206, 2014.

2. A. Kosterev, Y. Bakhirkin, R. Curl, and F. Tittel, "Quartz-enhanced photoacoustic spectroscopy," Optics Letters, vol. 27, pp. 1902-1904, 2002.

3. A. Elia, P. M. Lugará, C. Di Franco, and V. Spagnolo, "Photoacoustic techniques for trace gas sensing based on semiconductor laser sources," Sensors, vol. 9, no. 12, pp. 9616-9628, 2009.

4. A. A. Kosterev, F. K. Tittel, D. V. Serebryakov, A. L. Malinovsky, and I. V. Morozov, "Applications of quartz tuning forks in spectroscopic gas sensing," Review of Scientific Instruments, vol. 76, no. 4, p. 043105, 2005.

5. H. Zheng, X. Yin, L. Dong, H. Wu, X. Liu, W. Ma, L. Zhang, W. Yin, and S. Jia, "Multi-quartz enhanced photoacoustic spectroscopy with different acoustic microresonator configurations," Journal of Spectroscopy, vol. 2015, Article ID 218413, 6 pages, 2015.

6. P. Patimisco, A. Sampaolo, L. Dong, F. K. Tittel, and V. Spagnolo, "Recent advances in quartz enhanced photoacoustic sensing," Applied Physics Reviews, vol. 5, no. 1 , p. $011106,2018$.

7. A. A. Kosterev and J. H. Doty III, "Resonant optothermoacoustic detection: technique for measuring weak optical absorption by gases and micro-objects," Optics Letters, vol. 35, no. 21, pp. 3571 - 3573, 2010.

8. M. Spajer, B. Cavallier, S. Euphrasie, G. Matten, X. Vacheret, P. Vairac, D. Vernier, and A. Jalocha, "Thermoelastic investigation of a quartz tuning fork used in infrared spectroscopy," Applied Physics Letters, vol. 103, no. 20, p. 201111, 2013.

9. R. Curl, F. Capasso, C. Gmachl, A. Kosterev, B. McManus, R. Lewicki, M. Pusharsky, G. Wysocki, and F. Tittel, "Quantum cascade lasers in chemical physics," Chemical Physics Letters, vol. 487, pp. 1-18, 2010.

10. H. Zheng, L. Dong, H. Wu, X. Yin, L. Xiao, S. Jia, R. Curl, and F. Tittel, "Application of acoustic microresonators in quartz-enhanced photoacoustic spectroscopy for trace gas analysis," Chemical Physics Letters, vol. 691, pp. 462-472, 2018.

11. L. Dong, A. A. Kosterev, D. Thomazy, and F. K. Tittel, "QEPAS spectrophones: design, optimization, and performance," Appl. Phys. B, vol. 100, pp. 627-635, 2010.

12. Y. Liu, J. Chang, J. Lian, Z. Liu, Q. Wang, and Z. Qin, "Quartz-enhanced photoacoustic spectroscopy with right-angle prism," in Sensors, 2016.

13. L. Dong, H. Wu, H. Zheng, Y. Liu, X. Liu, W. Jiang, L. Zhang, W. Ma, W. Ren, W. Yin, S. Jia, and F. K. Tittel, "Double acoustic microresonator quartz-enhanced photoacoustic spectroscopy," Opt. Lett., vol. 39, no. 8, pp. 2479-2482, 2014.

14. N. Petra, J. Zweck, A. A. Kosterev, S. E. Minkoff, and D. Thomazy, "Theoretical analysis of a quartz-enhanced photoacoustic spectroscopy sensor," Applied Physics B, vol. 94, no. 4, pp. 673-680, 2009.

15. S. Firebaugh, E. Terray, and L. Dong, "Optimization of resonator radial dimensions for quartz enhanced photoacoustic spectroscopy systems," in Proc. SPIE 8600, Laser Resonators, Microresonators, and Beam Control $X V, 86001 S, 2013$.
16. G. Aoust, R. Levy, M. Raybaut, A. Godard, J.-M. Melkonian, and M. Lefebvre, "Theoretical analysis of a resonant quartz-enhanced photoacoustic spectroscopy sensor," Applied Physics B, vol. 123, no. 2, pp. 63:1-11, 2017.

17. J. H. Doty, A. A. Kosterev, and F. K. Tittel, "First experimental studies of the resonant optothermoacoustic detection technique," in CLEO/QELS: 2010 Laser Science to Photonic Applications, 2010, pp. 1-2.

18. P. Morse and K. Ingard, Theoretical Acoustics. McGrawHill, 1968.

19. J. Kaderli, J. Zweck, A. Safin, and S. Minkoff, "An analytic solution to the coupled pressure-temperature equations for modeling of photoacoustic trace gas sensors," Journal of Engineering Mathematics, vol. 103, no. 1, pp. 173-193, 2017.

20. A. Safin, S. E. Minkoff, and J. Zweck, "A preconditioned finite element solution of the coupled pressuretemperature equations used to model trace gas sensors," SIAM Journal on Scientific Computing, vol. 40, no. 5, pp. B1470-B1493, 2018.

21. B. Brennan, R. C. Kirby, J. Zweck, and S. E. Minkoff, "High-performance python-based simulations of pressure and temperature waves in a trace gas sensor," in Proceedings of PyHPC 2013: Python for high perfomance and scientific computing, 2013.

22. B. Brennan and R. C. Kirby, "Finite element approximation and preconditioners for a coupled thermal-acoustic model," Computers \&5 Mathematics with Applications, vol. 70, no. 10, pp. $2342-2354,2015$.

23. A. A. Kosterev and F. K. Tittel, "Ammonia detection by use of quartz-enhanced photoacoustic spectroscopy with a near-IR telecommunication diode laser," Appl. Opt., vol. 43, no. 33, pp. 6213-6217, 2004.

24. N. Petra, J. Zweck, S. E. Minkoff, A. A. Kosterev, and J. H. Doty III, "Modeling and design optimization of a resonant optothermoacoustic trace gas sensor," SIAM Journal on Applied Mathematics, vol. 71, no. 1, pp. 309$332,2011$.

25. A. Safin, "Modeling trace gas sensors with the coupled pressure-temperature equations," Ph.D. dissertation, The University of Texas at Dallas, 2018.

26. P. Heyliger, H. Ledbetter, and S. Kim, "Elastic constants of natural quartz," The Journal of the Acoustical Society of America, vol. 114, no. 2, pp. 644-650, 2003.

27. D. E. Carlson, Linear Thermoelasticity. Berlin, Heidelberg: Springer Berlin Heidelberg, 1973, pp. 297-345.

28. G. W. Stewart, "A krylov-schur algorithm for large eigenproblems," SIAM Journal on Matrix Analysis and Applications, vol. 23, no. 3, pp. 601-614, 2002.

29. V. Hernandez, J. E. Roman, and V. Vidal, "SLEPc: A scalable and flexible toolkit for the solution of eigenvalue problems," ACM Trans. Math. Software, vol. 31, no. 3, pp. 351-362, 2005.

30. P. R. Amestoy, I. S. Duff, J.-Y. L'Excellent, and J. Koster, "A fully asynchronous multifrontal solver using distributed dynamic scheduling," SIAM Journal on Matrix Analysis and Applications, vol. 23, no. 1, pp. 1541, 2001.

31. J. E. Roman, C. Campos, E. Romero, and A. Tomas, "SLEPc users manual," D. Sistemes Informàtics i Computació, Universitat Politècnica de València, Tech. Rep. DSIC-II/24/02 - Revision 3.9, 2018. 
32. A. Miklós, S. Schäfer, and P. Hess, "Photoacoustic spectroscopy, theory," in Encyclopedia of Spectroscopy and Spectrometry, J. C. Lindon, G. E. Tranter, and J. L. Holmes, Eds. Academic Press, 2000, vol. 3, pp. 18151822.

33. A. Siegman, Lasers. University Science Books, 1986.

34. J.-P. Berenger, "A perfectly matched layer for the absorption of electromagnetic waves," J. Comput. Phys., vol. 114 , no. 2, pp. 185-200, 1994.

35. W. C. Chew and W. H. Weedon, "A 3D perfectly matched medium from modified Maxwell's equations with stretched coordinates," Microwave and Optical Technology Letters, vol. 7, no. 13, pp. 599-604, 1994.

36. G. Aoust, R. Levy, B. Bourgeteau, and O. Le Traon, "Viscous damping on flexural mechanical resonators," Sensors and Actuators A: Physical, vol. 230, pp. 126$135,2015$.

37. I. N. Sneddon, The linear theory of thermoelasticity. Springer, 1974.

38. W. Bangerth, R. Hartmann, and G. Kanschat, "deal.II a general purpose object oriented finite element library," ACM Trans. Math. Softw., vol. 33, no. 4, pp. 24/1-24/27, 2007.

39. S. Balay, S. Abhyankar, M. F. Adams, J. Brown, P. Brune, K. Buschelman, L. Dalcin, V. Eijkhout, W. D. Gropp, D. Kaushik, M. G. Knepley, L. C. McInnes, K. Rupp, B. F. Smith, S. Zampini, H. Zhang, and H. Zhang, "PETSc users manual," Argonne National Laboratory, Tech. Rep. ANL-95/11 - Revision 3.7, 2016.

40. S. Balay, W. D. Gropp, L. C. McInnes, and B. F. Smith, "Efficient management of parallelism in object oriented numerical software libraries," in Modern Software Tools in Scientific Computing, E. Arge, A. M. Bruaset, and H. P. Langtangen, Eds. Birkhäuser Press, 1997, pp. 163-202.

41. C. Geuzaine and J.-F. Remacle, "Gmsh: A 3-D finite element mesh generator with built-in pre- and postprocessing facilities," International Journal for Numerical Methods in Engineering, vol. 79, no. 11, pp. 13091331, 2009.

42. C. Burstedde, L. C. Wilcox, and O. Ghattas, "p4est: Scalable algorithms for parallel adaptive mesh refinement on forests of octrees," SIAM Journal on Scientific Computing, vol. 33, no. 3, pp. 1103-1133, 2011.

43. M. Heroux, R. Bartlett, V. H. R. Hoekstra, J. Hu, T. Kolda, R. Lehoucq, K. Long, R. Pawlowski, E. Phipps, A. Salinger, H. Thornquist, R. Tuminaro, J. Willenbring, and A. Williams, "An Overview of Trilinos," Sandia National Laboratories, Tech. Rep. SAND2003-2927, 2003.

44. P. R. Amestoy, A. Guermouche, J.-Y. L'Excellent, and S. Pralet, "Hybrid scheduling for the parallel solution of linear systems," Parallel Computing, vol. 32, no. 2, pp. 136-156, 2006.

45. G. Gautschi, Piezoelectric Sensorics. Heidelberg: Springer, 2002.

46. S. L. Firebaugh, F. Roignant, and E. A. Terray, "Modeling the response of photoacoustic gas sensors," in Proceedings of the COMSOL Conference, 2009.

47. A. Kosterev, Y. Bakhirkin, F. Tittel, S. Blaser, Y. Bonetti, and L. Hvozdara, "Photoacoustic phase shift as a chemically selective spectroscopic parameter," $A p$ plied Physics B, vol. 78, no. 6, pp. 673-676, 2004. 